\title{
Heartbeat: improved quality of life and reduced healthcare utilisation after catheter ablation in patients with drug-resistant paroxysmal atrial fibrillation
}

The role of catheter ablation of atrial fibrillation (AF) in management of patients with paroxysmal AF is controversial. In this issue of Heart, Gupta and colleagues ${ }^{1}$ report data from a multicentre study of 329 consecutive patients with drug-refractory paroxysmal AF treated with AF ablation by pulmonary vein isolation guided by a standardised CLOSE (contiguous optimised lesions) protocol. Patient reported quality of life (QOL) measures showed significant improvement across all domains at 12 months. In addition, QOL improvement was associated with a lower AF burden, measured by ambulatory monitoring. Overall, cardiovascular hospitalisations decreased by $42 \%$ after AF ablation. Patients with the lowest QOL measures at baseline had the most improvement after AF ablation (figure 1).

In the accompanying editorial, Elvan ${ }^{2}$ comments: 'Significant reduction of the impact of AF on healthcare utilisation and improvement of QOL metrics should be regarded as important and patient-relevant healthcare values gained by catheter ablation of paroxysmal AF. Moreover, Gupta and colleagues ${ }^{1}$ report an inverse association between the extent of QOL improvement and residual $\mathrm{AF}$ burden post-ablation. These results emphasise the importance of incorporating AF-specific QOL metrics in AF ablation studies.' Ongoing innovations in approaches to AF ablation are discussed as well.

Identification of predictors of sudden cardiac death (SCD) at the population level are needed for prevention because up to $1 / 2$ of events occur in people with no prior history of heart disease. Ågesen and colleagues ${ }^{3}$ report the temporal trends in SCD in 14562 participants followed in the Copenhagen City Heart

Division of Cardiology, University of Washington, Seattle, Washington, USA

Correspondence to Professor Catherine M Otto, Division of Cardiology, University of Washington, Seattle, WA 98195, USA; cmotto@uw.edu

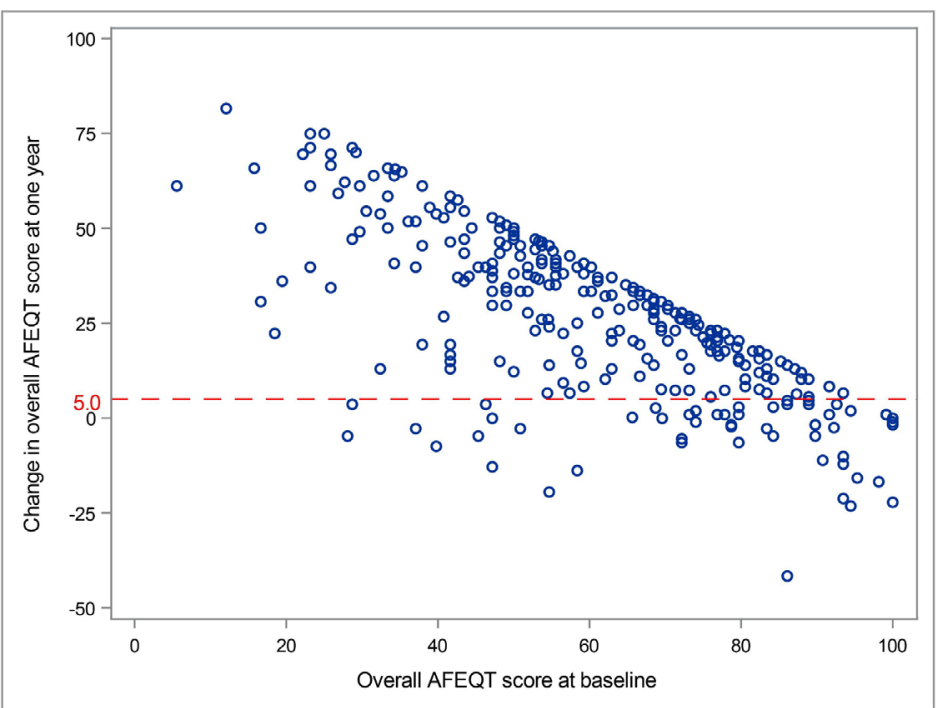

\begin{tabular}{|c|c|c|c|c|c|c|}
\hline $\begin{array}{l}\text { Baseline Overall } \\
\text { AFEQT Score }\end{array}$ & $\begin{array}{c}0-20 \\
(N=7)\end{array}$ & $\begin{array}{l}21-40 \\
(N=39)\end{array}$ & $\begin{array}{c}41-60 \\
(N=98)\end{array}$ & $\begin{array}{c}61-80 \\
(\mathrm{~N}=100)\end{array}$ & $\begin{array}{l}81-100 \\
(N=57)\end{array}$ & $\begin{array}{l}\text { Total } \\
(\mathrm{N}=301)\end{array}$ \\
\hline $\begin{array}{l}\text { Patients with } \\
\text { Gain } \geq 5 \text { points }\end{array}$ & $\begin{array}{c}7 \\
(100 \%)\end{array}$ & $\begin{array}{c}35 \\
(89.7 \%)\end{array}$ & $\begin{array}{c}91 \\
(92.9 \%)\end{array}$ & $\begin{array}{c}85 \\
(85 \%)\end{array}$ & $\begin{array}{c}28 \\
(49.1 \%)\end{array}$ & $\begin{array}{c}246 \\
(81.7 \%)\end{array}$ \\
\hline $\begin{array}{l}\text { Patients with Loss or } \\
\text { Gain }<5 \text { points }\end{array}$ & $\begin{array}{c}0 \\
(0.0 \%)\end{array}$ & $\begin{array}{c}4 \\
(10.3 \%)\end{array}$ & $\begin{array}{c}7 \\
(7.1 \%)\end{array}$ & $\begin{array}{c}15 \\
(15 \%)\end{array}$ & $\begin{array}{c}29 \\
(50.9 \%)\end{array}$ & $\begin{array}{c}55 \\
(18.3 \%)\end{array}$ \\
\hline
\end{tabular}

Figure 1 Atrial fibrillation effect on quality of life survey (AFEQT) change versus baseline score.



Figure 2 The incidence rate of sudden cardiac death from 1993 to 2016 per age group stratified by sex. Data are expressed as incidence rates and $95 \% \mathrm{Cls}$. PY, person-years. 


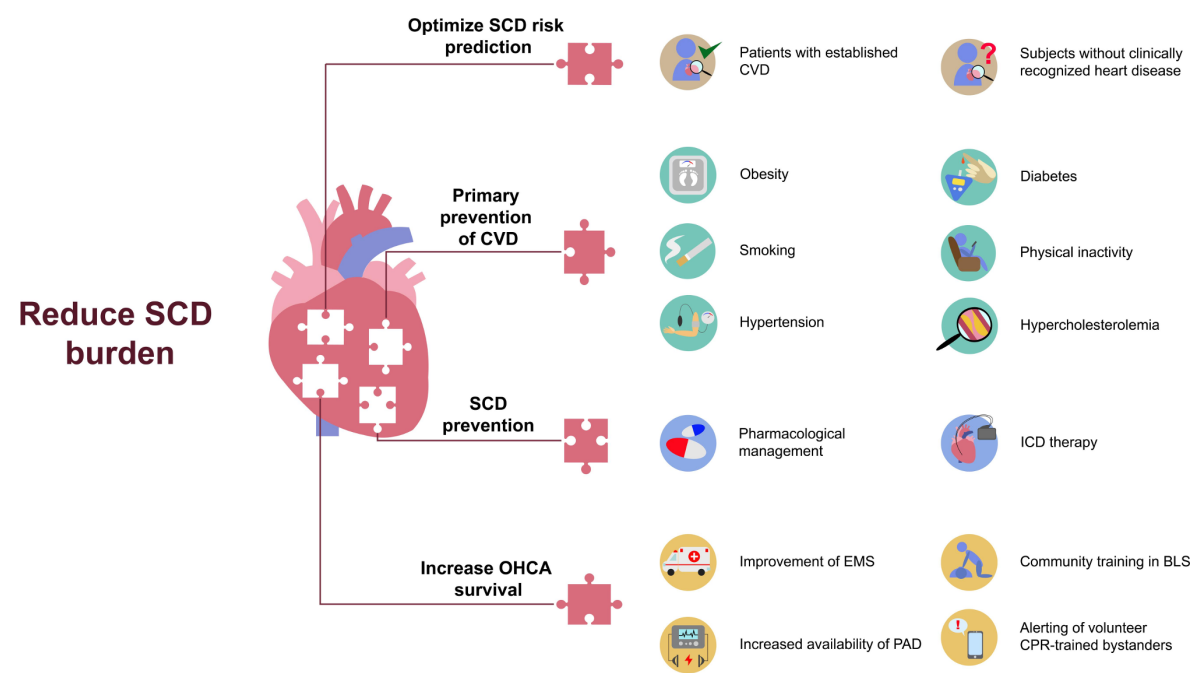

Figure 3 Priority areas and relevant actions needed to reduce the burden of SCD. BLS, basic life support; CPR, cardiopulmonary resuscitation; CVD, cardiovascular disease; EMS, emergency medical service; ICD, implantable cardioverter defibrillator; OHCA, out-of-hospital cardiac arrest; PAD, public access defibrillator; SCD, sudden cardiac death.

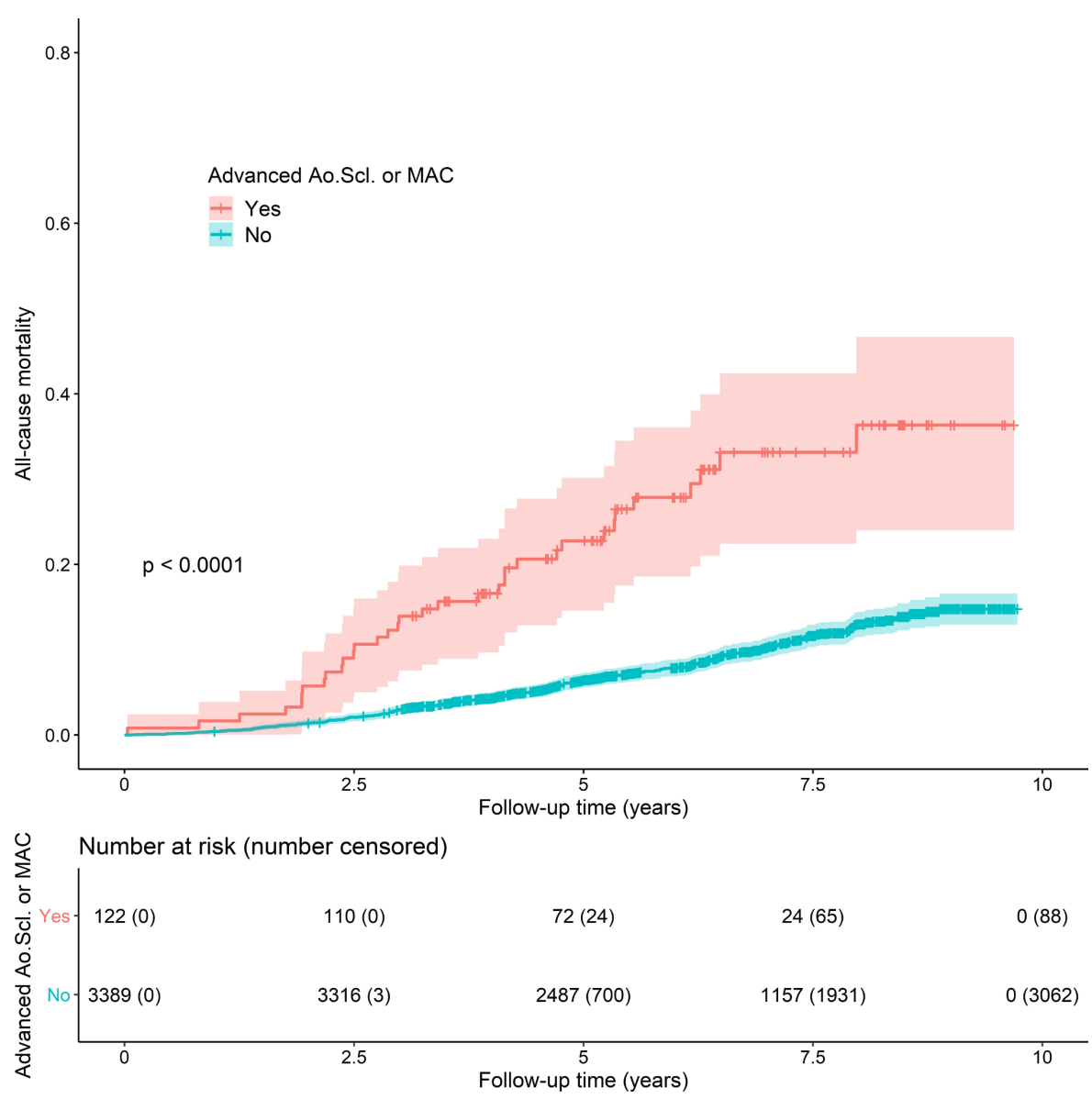

Figure 4 Kaplan-Meier curve demonstrating the unadjusted survival rates for people with advanced aortic sclerosis (Ao.Scl) or mitral annular calcification (MAC) compared with people with early or no disease. Participants are categorised as having advanced aortic sclerosis or mitral annular calcification (types of calcific valve disease without functional effect), irrespective of the presence of valvular heart disease. Advanced disease describes moderate or significant sclerosis or calcification, although without functional impact
Study from 1993 to 2016 . Of the 8394 deaths with full information, 1335 $(16 \%)$ were classified as SCD with a $41 \%$ decrease in SCD incident over the study period in persons aged 40-90 years (figure 2). There was a higher incidence of SCD in men, compared with women in those age 75 years or less with an incidence ratio of 1.99 (95\% CI 1.62 to 2.46 ) with SCD being the first known manifestation of cardiac disease in $50 \%$ of cases.

Tzeis urges in an editorial ${ }^{4}$ that: 'Further actions should aim to reduce the rate of SCD by focusing on two priority areas. The first one is prevention of cardiovascular disease by promoting the adoption of healthy lifestyle and behavioural habits and by implementing comprehensive intervention programmes to tackle cardiovascular risk factors. The second priority area is primary and secondary prevention of SCD' (figure 3).

The increasing recognition that some types of mild valve disease are associated with adverse clinical outcomes is highlighted in a study by Taylor and colleagues ${ }^{5}$ in this issue of Heart. In a population-based cohort from the OxVALVE (Oxford Valvular Heart Disease) study that included 3511 participants over age 65 years, advanced aortic valve sclerosis (present in $2.25 \%$ ) and advanced mitral annular calcification (present in 1.31\%) were associated with a higher risk of death (HR 2.05, 95\% CI 1.28 to 3.30 and HR 2.51 , 95\% CI 1.41 to 4.49 , respectively) (figure 4).

Iung and Bouleti comment ${ }^{6}$ that 'This analysis of the OxValve cohort suggests that more attention should be paid to the extent of the calcific valve lesion as assessed by echocardiography even at the early stages of valvular disease. Although this cannot translate in effective prevention measures at the present time, these findings further highlight the need for continuous research on the pathophysiology of calcific valve diseases, and the identification of metabolic pathways which may reduce the consequences of calcium deposits.'

A systematic review on patient preferences and values related to the choice of prosthetic valve for treatment of severe aortic stenosis provides useful insights and also underlines the need to more fully integrate the patient point of view into future clinical trial designs. ${ }^{7}$ Identifying the factors important to patients in shared decision making and involving patients in defining relevant 
Management of an individual with excessive LV trabeculation

Clinical history
Symptoms or clinical signs that warrant further investigation: heart failure, palpitation, syncope
Presentations suspicious for cardiomyopathy: stroke, TIA, systemic embolism, cardiac arrest
Factors potentially influencing expression of trabeculation: age, ethnicity, haemoglobinopathy, neuromuscular disorder,
mitochondrial cytopathy, athlete, late pregnancy
Family pedigree +/- results from first-degree family member screening
ECG
Assessment for abnormalities associated with myocardial disease: LBBB, T wave inversion, ST changes, pathologic Q
waves, ventricular pre-excitation, conduction disease, AV blocks, high ventricular ectopic burden, low ECG voltages,
atrial or ventricular arrhythmia
QRS duration $\geq 130$ ms influences cardiac device choice if indicated - CRT
Cardiac imaging
Assessment for regional wall motion abnormalities, wall thinning, thrombus, impaired LV systolic function, LVEF,
diastolic function, RV function, pulmonary hypertension, valvular heart disease, congenital heart disease, additional
features of DCM/HCM/RCM/AVC
CMR: LGE pattern suggestive of a non-ischaemic cardiomyopathy pattern, classical infarct or embolism
Consideration of gene testing according to family pedigree and genetic service policy
Treatment
For HFrEF (LVEF <40\%): ACE-I/ARB/ARNI, Beta-Blocker, MRA, Ivabradine, SGLT2i per heart failure guidelines
ICD/CRT based on NI-DCM primary and secondary prevention indications (including Lamin A/C mutation carriers)
Pre-pregnancy risk assessment and counseling, cardiac rehabilitation, monitoring for indications and contraindications
to cardiac transplantation
Anticoagulation for patients with AF and intracardiac thrombus. Consider with stroke, TIA and systemic embolism
presentations. Debatable on the grounds of LVSD or CHADS ${ }_{2}$ score $\geq 2$ without AF
Individuals with isolated excessive trabeculation and no other abnormalities can be considered for reassurance and
discharge from follow up as they may represent part of a wide spectrum of normal ventricular trabeculation

Figure 5 Management algorithm of individuals with excessive LV trabeculation. ACE-I, ACE inhibitor; $A F$, atrial fibrillation; $A R B$, angiotensin II receptor blocker; $A R N I$, angiotensin receptorneprilysin inhibitor; AVC, arrhythmogenic ventricular cardiomyopathy; $\mathrm{CHADS}_{2}$, congestive heart failure, hypertension, age, diabetes, stroke or transient ischaemic attack; CMR, cardiac magnetic resonance; CRT, cardiac resynchronisation therapy; DCM, dilated cardiomyopathy; HCM, hypertrophic cardiomyopathy; HFrEF, heart failure with reduced ejection fraction; ICD, implantable cardioverter-defibrillator; LBBB, left bundle branch block; LGE, late gadolinium enhancement; LV, left ventricular; LVEF, left ventricular ejection fraction; LVSD, left ventricular systolic dysfunction; MRA, mineralocorticoid receptor antagonist; NI-DCM, non-ischaemic dilated cardiomyopathy; $\mathrm{RCM}$, restrictive cardiomyopathy; RV, right ventricular; SGLT2i, sodium-glucose cotransporter 2 inhibitor; TIA, transient ischaemic attack.

outcomes is essential for ensuring that medical care meets patient needs.

The Education in Heart article in this issue reviews the causes, diagnosis and management of left ventricular noncompaction (figure 5). ${ }^{8}$
Funding The authors have not declared a specific grant for this research from any funding agency in the public, commercial or not-for-profit sectors.

Competing interests None declared.

Patient and public involvement Patients and/or the public were not involved in the design, or conduct, or reporting, or dissemination plans of this research.
Patient consent for publication Not required. Provenance and peer review Commissioned; internally peer reviewed.

(C) Author(s) (or their employer(s)) 2021. No commercial re-use. See rights and permissions. Published by BMJ.

\section{Check for updates}

To cite Otto CM. Heart 2021;107:1271-1273.

Heart 2021;107:1271-1273.

doi:10.1136/heartjnl-2021-320029

ORCID iD

Catherine M Otto http://orcid.org/0000-0002-05279392

\section{REFERENCES}

1 Gupta D, Vijgen J, Potter TD, et al. Quality of life and healthcare utilisation improvements after atrial fibrillation ablation. Heart 2021;107:1296-302.

2 Elvan A. It's all about improvement of quality of life and reduction of disease burden in atrial fibrillation ablation. Heart 2021;107:1274-5.

3 Ågesen FN, Lynge TH, Blanche P, et al. Temporal trends and sex differences in sudden cardiac death in the Copenhagen City heart study. Heart 2021;107:1303-9.

4 Tzeis S. Sudden cardiac death: we are doing well ... but we need to do better! Heart 2021;107:1276-7.

5 Taylor CJ, Ordóñez-Mena JM, Jones NR. Survival of people with valvular heart disease in a large, English community-based cohort study. Heart 2021;107:1336-43.

6 lung B, Bouleti C. Adverse cardiovascular disease outcomes in patients with aortic sclerosis and mitral annular calcification even when valve function is normal. Heart 2021;107:1280-1.

7 Heen AF, Lytvyn L, Shapiro M, et al. Patient values and preferences on valve replacement for aortic stenosis: a systematic review. Heart 2021;107:1289-95.

8 D'Silva A, Jensen B. Left ventricular non-compaction cardiomyopathy: how many needles in the haystack? Heart 2020:1344-52 